\title{
Alltagspraxis richterlicher Gesetzesbindung und ihre institutionellen Voraussetzungen
}

Um die „Rechtslage“ einzuschätzen, orientieren sich Juristen erstaunlicherweise immer noch an Gesetzestexten; gegebenenfalls ergänzt durch einen Blick in den einschlägigen Kommentar und, wie es scheint, entsprechend angeleiteter Subsumtion. Erstaunlich ist dies, weil angesichts der Befunde der Rechtstheorie schwerlich davon ausgegangen werden kann, dass unterschiedliche Rezipienten desselben Textes zu gleichen Ergebnissen kommen werden. Denn die Vorstellung, man ,subsumiere“ den Sachverhalt unter eine Rechtsnorm, wird spätestens seit den 60er Jahren des vorigen Jahrhunderts für naiv, simplifizierend oder sogar für völlig unangemessen gehalten. ${ }^{1}$ Dennoch reklamieren gerichtliche Entscheidungsbegründungen für sich in aller Regel eine so zwingende Eindeutigkeit, dass man meinen könnte, dies geschehe vor einem methodentheoretischen Horizont ungebrochener Begriffsjurisprudenz. Winfried Hassemer hat zu solchen Widersprüchen bereits 1972 bedauernd angemerkt, dass zwischen juristischer Praxis und ihrer Theorie leider kein merkbarer Rückkoppelungseffekt bestehe. ${ }^{2}$

\section{Der aktuelle Disput}

Mit gutem Grund nimmt die seit 2006 wieder aufgelebte Diskussion um die richterliche Gesetzesbindung ${ }^{3}$ diesen Widerspruch auf. Dabei wird aus einer eher demokratietheoretischen Perspektive das Prinzip der Gewaltenteilung mit der Frage ins Verhältnis gesetzt, ob sprachlich gefasste rechtliche Regeln richterliches Handeln leiten können und sollen. Auffallend ist, dass die aktuellen Stellungnahmen zwischen zwei Extremen schwanken: Einerseits wird die richterliche Bindung an legislativ vorgegebene Texte unbeirrt eingefordert, andererseits wird entschieden postuliert, gerade dies sei aufgrund des rechtskonstruktiven Aspekts jeder richterlichen Entscheidung geradezu ein Ding der Unmöglichkeit.

Etwas überpointiert könnte man letztere Position wie folgt paraphrasieren: Rechtstexte können in rechtliches Handeln gar nicht anders umgesetzt werden als durch rechtsschöpferische Dezision des entscheidenden Gerichts. Denn zum einen ist Sprache zu vage und unbestimmt, zum anderen lässt die ungeregelte juristische Methodenvielfalt keine zwingende Selektion von Handlungsmöglichkeiten zu. Aus dieser Perspektive wird Recht tendenziell auf den prozeduralen Charakter der Prozessabläufe, die Auslese

1 Statt vieler F. Müller, Juristische Methodik, 6. A. 1995, S. 79 ff., $156 \mathrm{f}$.

2 Juristische Argumentationstheorie und juristische Didaktik, in: Hans Albert et al., Rechtstheorie als Grundlagenwissenschaft der Rechtswissenschaft, 1972, S. 467 (472).

3 G. Hirsch, ZRP 2006, S. 161; FAZ v. 30.4.2007, S. 8; Grasnick, FAZ v. 4.1.2008, S. 36; Möllers, FAZ v. 26.10.2006, S. 37; Jeep, FAZ v. 29.11.2006, S. 21; Ullmann, FAZ v. 29.11.2006, S. 21; Kloke, FAZ v. 29.11.2006, S. 21; Rüthers, JZ 2006, S. 53; ders., FAZ v. 27.12.2006, S. 31; ders., ZRP 2008, S. 48; ders., JZ 2008, S. 446; G. Roellecke, FAZ v. 30.1.2007, S. 34; D. Simon, Myops 1/2007, S. 21; Hassemer, ZRP 2007, 213; M. Kriele, ZRP 2008, S. 51. 
derjenigen, die entscheiden, und den legitimatorischen Anstrich reduziert, den die Berufung auf Rechtsquellen ermöglicht. Wem eine solch schmale Basis rechtlicher Bindung zu unbestimmt und damit in demokratietheoretischer Hinsicht problematisch erscheint, der versucht die richterliche Gesetzesbindung durch Methodenkritik zu retten: So will etwa Rüthers den derzeit praktizierten Methodenpluralismus durch eine strikte Einschränkung und Stufenfolge zulässiger Auslegung disziplinieren. ${ }^{4}$ Vorschläge dieser Art sind nicht ganz neu. ${ }^{5}$ Wer Textverstehen jedoch für einen hermeneutischen Vorgang hält, der keine objektive überindividuelle Leistung darstellt, sondern sich entlang auch subjektiv geprägter Vorverständnisse vollzieht, ${ }^{6}$ wird bezweifeln, dass sich richterliche Dezision auf diese Weise stärker an Gesetzestexte binden lässt.

\section{Gesetzesbindung im forensischen Alltag}

Indes scheint es um das Anliegen richterlicher Gesetzesbindung gar nicht so schlecht bestellt zu sein, wenn man sich die forensische Alltagspraxis aus einer eher rechtssoziologischen Perspektive vor Augen hält und dabei den Fokus stärker auf die Instanzgerichte lenkt. Auch dieser Blickwinkel ändert allerdings nichts daran, dass jede Vorstellung richterlicher Gesetzesbindung voraussetzt, dass zwischen Gesetzestext, Textverständnis und richterlicher Entscheidung ein Zusammenhang besteht, der deutlich über den legitimatorischen Anstrich des für richtig Befundenen hinausgeht. Die zentrale Frage lautet also, ob sich Mechanismen eines solchen Zusammenhangs feststellen lassen. Nicht von ungefähr wird auch von rechtssoziologischer Seite teilweise nachdrücklich bestritten, dass gerichtliche Begründungen den handlungsleitenden Motiven bei Herstellung der Entscheidung entsprechen. ${ }^{7}$ Daran ist jedenfalls richtig, dass zwischen Herstellung und Darstellung richterlicher Urteile sorgfältig zu unterscheiden ist. ${ }^{8}$ Aber selbst methodenkritisch Gestimmte werden einräumen, dass sich längst nicht jede mögliche Entscheidung gleich gut begründen lässt. Manche lässt sich gar nicht begründen oder nur mit erheblichem Aufwand und deutlich geringeren Chancen, professionsintern auf Zustimmung zu stoßen. Wer forensisch arbeitet, für den konstituiert diese Erfahrung geradezu einen erheblichen Teil der juristischen Alltagspraxis.

\section{Präferenzen für kommunikative Gemeinsamkeiten}

Versuchen wir es also probeweise mit der Hypothese, dass es für Juristinnen und Juristen in den meisten Fällen einen Fundus gemeinsam geteilter Präferenzen für bestimmte

4 Rüthers, ZRP 2008, S. 48.

5 Nachweise bei M. Deckert, Folgenorientierung in der Rechtsanwendung, 1995, S. 39 f.

6 Grundlegend hierzu J. Esser, Vorverständnis und Methodenwahl in der Rechtsfindung, 1972, S. $136 \mathrm{ff}$. und passim.

7 Exemplarisch Rottleuthner, Richterliches Handeln, 1973, S. 11 ff., 28 ff., 57, 61 ff.

8 Luhmann, Recht und Automatisation in der öffentlichen Verwaltung, 1966, S. 51; Rottleuthner (o. Fußn. 7), S. 63; ähnlich differenzieren J. Esser (o. Fußn. 6), S. 7, 112 ff.; Jost, Soziologische Feststellungen in der Rechtsprechung des Bundesgerichtshofs in Zivilsachen, 1979, S. 25 f.; H. A. Hesse, JZ 1996, S. 449 (453 f.); Alexy, Theorie der Grundrechte, 1986, S. 144; ders., Theorie der juristischen Argumentation, 1991, S. 282; Kriele, ZRP 2008, S. 51 (52). 
Begründungen und Begründungsformen gibt. Sollte dies richtig sein, dann muss man wohl auch davon ausgehen, dass die Alternativen richtigen Begründens die Möglichkeiten rechtlichen Entscheidens erheblich limitieren. Dies gilt vielleicht nicht für jeden Fall. Denn in den so genannten ,hard cases“ mögen sich Entscheidungen durchsetzen, die von solchen Präferenzen abweichen. Jedenfalls aber gilt es für den Regelfall des instanzgerichtlichen Alltags, der für die Rechtssicherheit und die Erwartungsbildung der Nachfrager rechtlicher Entscheidungen zentral ist. Vor diesem Hintergrund ist der Hinweis auf die faktisch vergleichsweise großen Freiheiten der Ober- und der Verfassungsgerichte möglicherweise nicht geeignet, die Frage richterlicher Gesetzesbindung für das gesamte Rechtssystem zu beantworten.

Wer methodenkritisch reflektiert, dem wird die oben gewählte Formulierung ,gut begründen" wenig zusagen. In der Tat ist es eine zentrale Frage, ob und wie sich unsere Alltagserfahrung, dass es mehr oder weniger gute Begründungen gibt, wissenschaftlich fassen lässt. Mit der vagen Berufung auf Evidenz ist es nicht getan. Juristische Begründungspräferenzen rätselhaften Konsensen oder einer vereinigenden Kraft der Sprache geschuldet zu sehen, kann mit Blick auf die Ergebnisse der sprachtheoretisch angeleiteten Rechtstheorie wenig befriedigen. Die juristische Argumentationstheorie beantwortet die Frage allenfalls teilweise, weil sie die Güte von Argumenten wägt und beschreibt, sich aber nicht damit beschäftigt, welche Mechanismen empirisch dazu motivieren, ,guten“ oder auch nur bestimmten Argumenten mehr als anderen zu folgen.

Besser lässt sich die Bildung juristischer Begründungspräferenzen in soziologischen Kategorien fassen. Solche Präferenzen sind ein sehr voraussetzungsvolles Phänomen, das auf einer Vielzahl ineinandergreifender und komplexer sozialer Mechanismen beruht. ${ }^{9} \mathrm{Zu}$ nennen sind hier zunächst ähnliche Ausbildungserfahrungen und die in vielem ähnliche Alltagspraxis, die unter Juristen konvergierende Präferenzen sichern, Rechtstexte in ähnlicher Weise zu verstehen und etwaige Differenzen stets durch Rückgriff auf die nächste gemeinsame Präferenz auszutragen. Vermittelt über institutionell gesicherte Abnahmezwänge, vor allem den Rechtsweg und das kollegialgerichtliche Zusammenwirken, verstärken diese Pfadbahnungsmechanismen Begründungslasten für ungewöhnliche, seltene und neue Argumentationen. All diese Mechanismen konstituieren einen komplexen und anspruchsvollen Fundus kommunikativer Gemeinsamkeiten der Professionsangehörigen der juristischen Sprachgemeinschaft.

\section{Sprachliche Interaktion}

Aus sprachkritischer Perspektive könnte man einwenden, dass solche kommunikativen Gemeinsamkeiten keinesfalls als fester Konsens existieren, weil Sprache vage und mehrdeutig ist. Zudem beruht Verstehen immer auch auf subjektiver Prägung und hängt von der konkreten Sprachsituation ab. ${ }^{10}$ Niemand weiß sicher, wie der andere eine Äußerung versteht. Jeder an sprachlicher Interaktion Beteiligte handelt jedoch auf Basis

9 Mehr als eine knappe Skizze ist an dieser Stelle nicht möglich. Eingehend hierzu Maitra, Regeln und Prinzipien, 2006, S. 89 ff.

10 Hunde lachen nicht. Aber, um ein Beispiel der Linguistik aufzugreifen, als Antwort auf die Frage, wo das Steak geblieben ist, wird der Hinweis auf einen lächelnden Hund situationsbezogen verstanden. 
erfahrungsgeprägter Vermutungen, wie bestimmte Zeichenfolgen, etwa die eigene Aussage oder Äußerungen Dritter von anderen verstanden werden. Ein auf intersubjektiven Verstehensprozessen basierendes Modell kann daher nicht mit Gewissheiten, sondern lediglich mit Wahrscheinlichkeiten und Unwahrscheinlichkeiten arbeiten. $\mathrm{Zu}$ solchen Wahrscheinlich- und Unwahrscheinlichkeiten gelangt man, weil sprachliche Interaktion stets auf den kommunikativen Vergangenheiten der Interagierenden beruht, die nie nur individuelle, sondern stets auch soziale sind. Ähnliche Vergangenheiten und gleichläufige Erfahrungen, die in ähnlichen Situationskontexten gemacht werden und als Erfahrungshorizonte Wahrnehmung und Weltwissen der Akteure prägen, führen zwar nicht zu einer starr fixierbaren Übereinstimmung von individueller Wahrnehmung, Zeichenverwendung und Sinnzuordung. Sie erhöhen aber die Wahrscheinlichkeit, dass die Verständnisse der Mitglieder einer professionellen Sprachgemeinschaft aufgrund von bereits einmal in Teilen ähnlich erlebten Situationen konvergieren. Das professionelle Erlernen sprachlicher Konventionen, Redewendungen, pragmatischer Routinen, geht mit spezifischen Vorstellungen dessen, „was typisch ist“, einher. ${ }^{11}$

In diesem approximativ eingeschränkten Sinn konstituiert sich die juristische Sprachgemeinschaft geradezu durch gemeinsame kommunikative Vergangenheiten: Wer im konkreten Fall ein anderes Textverständnis geltend machen will, muss sich in kommunikative Distanz zu diesen Gemeinsamkeiten begeben: Will er die Chancen auf Abnahme seiner Begründung (und damit auch seiner Entscheidung) wahren oder steigern, dann muss er durch Rekurs auf andere gemeinsame Textverständnisse argumentativ nachweisen, dass sich sein Textverständnis in das Gesamtensemble dogmatischer und präjudizieller Vorgaben gut einpasst, und zwar besser als andere Verständnisse, insbesondere die bisher dominierende Lösung. Damit legitimiert die Berufung auf h.M. und Präjudizien zugleich „Dissens und das Sich-Verlassen auf Konsens“"12 - mit einer Präferenz für den status quo des aktuell hegemonialen Textverständnisses und damit: einer Einschränkung von Entscheidungsspielräumen.

Kommunikative Distanz wird also eingeholt durch Rekurs auf kommunikative Gemeinsamkeiten. Man kann als Anwalt oder als Instanzgericht die eigene Rechtsauffassung durch die herrschende Meinung und die obergerichtliche Judikatur rechtfertigen. Wer aber eine damit nicht begründbare Entscheidung wünscht oder rechtfertigen will, der muss weiter ausholen und die eingefahrenen Argumentationsbahnen verlassen. ${ }^{13}$ Dies kostet Zeit und Arbeit, beides Ressourcen, die insbesondere im forensischen Alltag äußerst knapp sind. Außerdem ist es tendenziell unwahrscheinlicher, dass das Gericht oder die nächste Instanz neuen Textverständnissen und damit Begründungsmöglichkeiten folgen, als wenn man sich auf die herrschende Meinung oder ein oberinstanzliches Präjudiz berufen kann. Abweichen von Vorgegebenem wirkt zudem emotional beunruhigend, zumal dies keine Tugend ist, die in der den professionellen juristischen Ha-

11 Ähnl. Sobota, Stimmigkeit als Rechtsstruktur, in: ARSP 1991, S. 243 (251 ff.), im Anschluss an den britischen Rechtstheoretiker Bernard S. Jackson; grundlegend zum typisierenden, d.h. an Analogien orientierten Denken Bernhard Waldenfels, Ordnung im Zwielicht, 1987, S. $51 \mathrm{ff}$., bes. $63 \mathrm{ff}$.

12 N. Luhmann, Rechtssoziologie, 3. A. 1987, S. 289.

13 Ähnl. zum Prozess des Abgleichens mit Präjudizien Kriele, ZRP 2008, S. 51 (52). 
bitus formenden Ausbildung sonderlich geschult wird. ${ }^{14}$ All dies erzwingt insbesondere innerhalb des Rechtsstabs einen großen Bedarf an kurzen, routineförmigen Begründungsmöglichkeiten.

\section{Routinemäßiges Begründen entlang von Konditionalprogrammen}

Routinemäßiges Begründen wird im Wesentlichen dadurch ermöglicht, dass weite Rechtsgebiete durch Regeln, verstanden als Konditionalprogramme, dominiert werden. Sowohl Luhmann als auch Alexy haben diesen die forensische Alltagspraxis prägenden Normtyp beschrieben: ${ }^{15}$ Konditionalprogramme wie Regeln sind Normkonstrukte, die festlegen, dass bestimmte Ursachen entlang eines strikten Wenn/Dann-Schemas bestimmte Konsequenzen zur Folge haben sollen, also die klassische Tatbestands-/Rechtsfolge-Struktur aufweisen. Während die Wenn-Komponente, der Tatbestand, die Ausfilterung der entscheidungsrelevanten Informationen ermöglicht, ordnet die DannKomponente verbindlich eine bestimmte Konsequenz an. Letztere kann in einer Rechtsfolge bestehen oder in einer rechtsinternen Verknüpfungsanordnung, durch die Voraussetzungen oder Folgen der in Rede stehenden Kernvorschrift spezifiziert, erweitert oder eingeschränkt werden. ${ }^{16}$ Daraus ergeben sich komplexe Netzstrukturen. Denn der Obersatz, der gebildet wird, um die Entscheidung als Subsumtion darzustellen, ist meist eine Vernetzung verschiedener Einzelnormen. Diese sind miteinander durch Rechtsbegriffe, die man als Knotenpunkte ${ }^{17}$ oder Schaltstellen bezeichnen kann, verknüpft. Vom Knotenpunkt „fahrlässig“ im Normtext des § 823 I BGB gelangt man zur Legaldefinition des $§ 276$ II BGB, von dort zu den in Rechtsprechung und Lehre vorgenommenen Präzisierungsversuchen und Kasuistiken. Das Wissen um solche Netzstrukturen und die Fähigkeit, sie schnell und umstandslos zu erfassen, machen einen wesentlichen Teil juristischer Professionalität aus.

Strukturen in Konditionalform eignen sich besonders gut, schnell, also mit geringstmöglichem Aufwand an Zeit und Arbeit Entscheidungen so zu begründen, dass eine Abnahme durch die meist juristisch vertretenen Parteien, höhere Instanzen und die Rechtswissenschaft wahrscheinlich erscheint. Der Raum der Darstellungsmöglichkeiten (und damit zugleich der Raum möglicher Entscheidungen) wird ausgelotet, indem er in kleine gedankliche Elemente zerlegt wird, die jeweils durch Vergleich mit vorhandenen Interpretationen auf ihre Möglichkeiten für Konsens und Dissens hin befragt und verglichen werden. ${ }^{18}$ Jeder Knotenpunkt kann als Relevanzkriterium einer Konditionalnorm zum Problem werden, das dann durch Begriffsdefinitionen ,gelöst“ wird. Etwaige Problemfälle erschüttern nicht das ganze Ensemble der in Konditionalform kondensierten Falllösungserfahrungen, sondern nur Einzelelemente. Selbst an solchen

14 Vgl. D. Fabricius, Anpassungs- und Abwehrmechanismen, in: Greive (Hrsg.), Die Rolle der Richter und Richterinnen zwischen Rechtsprechung und Politik, 1995, S. 71 (76 ff., 84); ders., Selbst-Gerechtigkeit, 1996, S. 120 ff.; Maitra (o. Fußn. 9), S. 153 ff.

15 Luhmann (o. Fußn. 12), S. 88; R. Alexy, Theorie der Grundrechte, 1986, S. 76.

16 Vgl. Lübbe-Wolf, Rechtsfolgen und Realfolgen, 1981, S. 26 ff., $40 \mathrm{ff}$.

17 So Lübbe-Wolf (o. Fußn. 16), S. $40 \mathrm{ff}$.

18 Vgl. Kißler, Recht und Gesellschaft, 1984, S. 115; Luhmann (o. Fußn. 12), S. 285 ff.; J. Es$\operatorname{ser}$ (o. Fußn. 6), S. 33. 
Punkten werden Präjudizien und der Verweis auf eine herrschende Meinung genutzt, um die eigene Argumentation abzukürzen und die Chance für Folgebereitschaft und Abnahme zu vergrößern.

\section{Hard Cases}

Diese die Stabilität des Rechts garantierenden Mechanismen werden insbesondere in den so genannten „hard cases“ relativiert. Charakteristisch für solche Fälle ist, dass außerhalb des Rechts kommunizierte Rationalitäten, Problembewertungen und Argumente moralischer, politischer oder ökonomischer Provenienz mit Nachdruck solche Entscheidungsvarianten nahelegen, die sich nicht innerhalb der Bandbreite des bislang rechtlich Begründbaren rechtfertigen lassen. Daraus ergeben sich Entscheidungssituationen, die aufgrund ihrer emotionalen Intensität und des daraus resultierenden Beunruhigungsgehalts eine Form des ,mental switching "19 nahelegen. Man überprüft, ob der bisherige, routinegesicherte Alternativenraum erweitert werden kann. Solche Entscheidungssituationen fördern neue und häufig als Bruch mit tradierten Begründungskonventionen wahrgenommene Lösungen und deren Abnahme. Werden in den jeweiligen Begründungen die darin aufscheinenden, bislang rechtsexternen Wertungen explizit thematisiert, so öffnet sich an diesem Punkt rechtliche Kommunikation für außerrechtliche Argumente. Diese werden durch rekursive Bezugnahmen dann ihrerseits Bestandteil rechtsspezifischer Texttraditionen. Einbruchstellen solcher teilweise abrupt erfolgenden Brüche mit tradierten Textverständnissen sind insbesondere Generalklauseln und prinzipienorientierte Argumentationsformen. Topische Argumente, Interessen- und Prinzipienabwägungen sowie die Ausfüllung von Blankettnormen erscheinen aus juristischer Sicht beliebiger als Konditionalprogramme. Sie erleichtern die Aufnahme rechtsextern kommunzierter Argumente, Rationalitäten, Bewertungspräferenzen in den rechtlichen Diskurs. Damit laufen sie spezifisch juristischen Routinen, die sich primär an den Eigenwerten professsionstypischen Textverstehens orientieren, sowie Rechtssicherheit und Gleichbehandlung tendenziell zuwider.

Im juristischen Alltag jedoch überfordern solche weichen Rechtsformen mit inakzeptablen Rationalitätszumutungen sowie Abnahmeungewissheiten, erzeugen individuell wie im Rechtssystem Unruhe und Unsicherheit. Zudem gehen sie zu Lasten knapper Ressourcen (Zeit und Arbeit). Über kurz oder lang werden sie daher wieder durch Präjudizien, Fallgruppenbildung und Dogmatisierungsbestrebungen routinisiert. ${ }^{20}$ Es bildet sich eine juristische Eigenlogik aus, die sich - bis auf weiteres - gegen Sachargumente und Bewertungen moralischer, wirtschaftlicher oder politischer Provenienz abschottet. Selbst in Bereichen, die stärker durch weiche Normen, also Zweckprogramme, Generalklauseln und Prinzipien, dominiert werden, weist die Rechtspraxis eine

19 Zu dem der Entscheidungstheorie entlehnten Begriff vgl. Birger P. Priddat, Risiko, Ungewißheit und Neues: Epistemologische Probleme ökonomischer Entscheidungsbildung, in: Banse (Hrsg.), Risikoforschung zwischen Disziplinarität und Interdisziplinarität, 1996, S. 105 ff. (117).

20 Solche Prozesse lassen sich etwa am Beispiel der Persönlichkeitsrechtsentwicklung nachweisen; vgl. Maitra (o. Fußn. 9), S. 285 ff. 
starke Tendenz auf, diese einer Routinisierung zugänglich zu machen, indem sie durch Präjudizien und Fallgruppenbildungen herunterkonditionalisiert werden.

\section{Entscheidungsroutinisierung durch Limitierung der Darstellungsmöglichkeiten}

Das in soziologischer Hinsicht interessante Phänomen liegt in folgendem: Die primäre richterliche Pflicht, Streitfälle verbindlich zu entscheiden, läuft im Grunde jeder Routine und Regelhaftigkeit zuwider. Entscheidungen eignet stets ein starkes Element der Singulariät. Die Funktionalität von Regeln und Routinen aber liegt gerade darin, die singuläre Kontingenz von Entscheidungen auszumerzen und damit gesellschaftliche Komplexität sowie den durch zeitlich ungebremstes Räsonieren verursachten Ressourcenverbrauch zu reduzieren. Man muss nicht die Vielzahl aller möglichen Entscheidungskriterien abarbeiten, sondern kann sich weitgehend damit begnügen, Ähnlichkeiten zu vorhergehenden Fallentscheidungen abzugleichen. Da jedoch jeder Fall als Streitfall signalisiert, dass die einschlägigen Routinen, Regeln und Konventionen konkret in Zweifel gezogen wurden, können Begründungsroutinen das dezisionistische Element von Gerichtsentscheidungen nicht vollständig ersetzen. Singuläre Entscheidungen an Begründungsroutinen zu binden, kann daher stets nur in Näherungen gelingen. Begründungsroutinen reduzieren nur den Alternativenhorizont und damit das Ausmaß erforderlichen Entscheidens. Dieser Alternativenhorizont aber lässt sich - und genau dies macht seine Stabilität aus - nur um den Preis der oben skizzierten Kostenlast erweitern.

\section{Rechtspolitische Konsequenzen}

Hält man vorstehende Skizze für zutreffend, dann stellt sich die Frage nach der richterlichen Gesetzesbindung weniger auf dogmatischer oder rechtstheoretischer Ebene, sondern in ganz handgreiflicher rechtspolitischer Hinsicht. Denn die beschriebenen Mechanismen haben institutionelle Voraussetzungen, die aufgrund vergangener Reformen wie aktueller Reformbestrebungen gefährdet sind. An höchstrichterlicher Rechtssetzung verzweifelt und entzündet sich die Debatte um die richterliche Gesetzesbindung im Grunde nur, weil dort offensichtlich wird, was insgesamt gilt: In gleichem Maße, wie Abnahmezwänge abnehmen, wächst die Freiheit richterlicher Dezision. Diesem Phänomen durch Forderungen an eine andere, bessere juristische Methodik begegnen zu wollen, erscheint ein wenig hilflos. Denn schließlich sind es die Richterinnen und Richter selbst, die in letzter Instanz entscheiden, nach welcher Methodik sie begründen. Solange es jedoch nur die obersten Bundesgerichte und die Verfassungsgerichte sind, die solche Freiheiten von übergeordneter Kontrolle weitgehend unbeeinträchtigt genießen, muss man damit wohl leben. Sicher wäre mancher höchstrichterlichen Urteilsbegründung eine sorgfältigere Auseinandersetzung mit anderen Meinungen und etwas mehr Wertschätzung rechtswissenschaftlicher Äußerungen zu wünschen. Doch ist es nun einmal so: Irgendwann muss jemand letztverbindlich entscheiden. Das bedingt 
zwangsläufig wesentlich größere Freiheiten als jene, über die Richter der Vorinstanzen verfügen. $^{21}$

Was aber fatal ist und stets mit einer Einbuße an Rechtssicherheit, Erwartungsbildung und eben Gesetzesbindung bezahlt wird, ist die Tendenz, diesen Ausnahmefall am Ende eines in der Regel dreigliedrigen Instanzenwegs in einer immer größeren Vielzahl von Konstellationen zum Regelfall des gerichtlichen Alltags zu machen, indem man den Instanzenweg sowie Rechtsmittel beschneidet, Einzelrichter an die Stelle von Kollegialgerichten setzt und Richter der gleichen Instanzenebene über die mögliche Befangenheit ihres potentiell den gleichen Mittagstisch teilenden Kollegen entscheiden lässt. ${ }^{22}$ Die Gehörsrüge, über die der gerügte Richter selbst befindet ( $\$ 321$ a ZPO), ist der augenscheinlichste Ausdruck solcher Praxisferne politischer Entscheidungsträger, die jeder Psychologie und Menschenkenntnis Hohn spricht. ${ }^{23}$ Reformen mit dem auf Personaleinsparung zielenden Rotstift zu schreiben, mag partiell die Justizkassen entlasten. Aber die Allgemeinheit bezahlt teuer für diesen Willkürentscheidungen tendenziell Vorschub leistenden Unsinn. Das solche Maßnahmen legitimierende Zerrbild, Rechtsmittel dienten vor allem den Interessen geldgieriger Anwälte und unbelehrbarer Streithansel, überdeckt, dass der mehrstufige Instanzenzug eine zentrale Funktionsbedingung unseres Rechtssystems ist. Vor allem die durch ihn institutionell abgesicherten Abnahmeerfordernisse sind es, die richterlichen Versuchungen entgegenstehen, entlang subjektiver Präferenzen und damit möglichst frei von rechtlicher Bindung zu entscheiden. Richterliche Gesetzesbindung, verstanden als eine Präferenz für Begründungen, die sich im gesicherten Rahmen juristisch tradierter kommunikativer Gemeinsamkeiten halten, ist nicht billig zu haben. Denn sie setzt voraus, dass die sozialen Mechanismen, die eine solche Präferenz erzwingen, nicht unterminiert werden. Seitens der Politik wird teilweise mit erschreckender Praxisferne verkannt, dass gutes Recht nicht allein eine Frage (einzel-)richterlicher Kompetenz und Hinweistechnik ist. Letztlich ist es vor allem der durch Instanzenzug und Kollegialgerichte wirkende sanfte Zwang, konsistent und tendenziell konservativ an den kommunikativen Gemeinsamkeiten rechtlicher Diskurse orientiert zu begründen, der Willkür verhindert und Rechtssicherheit gewährleistet. Damit sichern die Garantie eines umfassenden Instanzenwegs und der Grundsatz, dass Entscheidungen durch möglichst unabhängige Dritte zu überprüfen sind, nicht allein individuelle Rechtsschutzinteressen. Vielmehr sind sie konstitutiv für die Fähigkeit des Rechts, flexibel auf äußere Anforderungen zu reagieren, ohne an Stabilität zu verlieren.

21 Darüber eine halbpolitische Instanz wie das Bundesverfassungsgericht zu setzen, die de facto recht frei entscheidet, ob sie Verfassungsbeschwerden annimmt, ist nachgerade ein genialer Wurf. Denn damit bleibt auch höchsten Instanzen stets ein Rest disziplinierender Unsicherheit, der allzu großen Freiheiten Grenzen setzt.

22 Über Ablehnungsgesuche wegen Besorgnis der Befangenheit entscheidet seit der Zivilprozessreform „ein anderer Richter des Amtsgerichts“ (§ 45 Abs. 2 S. 1 ZPO); krit. E. Schneider, ZAP Nr. 12/2005, Fach 13, S. 1299.

23 Vgl. E. Schneider, MDR 2006, S. 969 (971 f.). 